\title{
Thermodynamics of the Ternary System: Water-Sodium Chloride-Barium Chloride at $25{ }^{\circ} \mathrm{C}$
}

\author{
R. A. Robinson and V. E. Bower
}

\begin{abstract}
(September 15, 1964)
Isopiestic vapor pressure measurements have been made on the ternary system watersodium chloride-barium chloride at $25{ }^{\circ} \mathrm{C}$. From these data the activity coefficient of each salt in the presence of the other has been calculated. The rule that the logarithm of the activity coefficient of one salt (which is equivalent to its chemical potential) varies linearly with the concentration of the other salt has been investigated. It is shown that this rule is at least approximately valid with the condition usually assumed, namely, that the total ionic strength is kept constant. It is shown, moreover, to be valid for another condition, namely, that the total concentration of ions is kept constant. This latter condition is more suited to isopiestic studies. Finally, the calculation of mutual solubilities from isopiestic data is described.
\end{abstract}

\section{Introduction}

Systems of the type $\mathrm{H}_{2} \mathrm{O}-\mathrm{HCl}-\mathrm{MCl}$, where $\mathrm{M}$ designates a univalent cation, have been studied extensively [1,2], ${ }^{1}$ using concentration cells with hydrogen and silver-silver chloride electrodes. Such cells are admirably suited to the determination of the chemical potential and activity coefficient of hydrochloric acid in the presence of added chloride and to a study of the variation of the activity coefficient of hydrochloric acid under selected conditions. Thus, one may ask how the activity coefficient of hydrochloric acid varies if the concentration of acid is kept constant and sodium chloride is added in increasing amounts. Or one may ask how the activity coefficient of the acid would vary if both the acid and the salt concentration were allowed to change subject to a restraining condition; such a condition might be that the total ionic strength remained constant.

Questions of this nature have been answered in considerable detail by Harned and his colleagues [1]. Moreover, studies have been extended to systems in which the added chloride is that of a polyvalent cation, for example, barium or strontium chloride $[3,4]$ or cadmium chloride [5]. Systems have also been studied in which there is no common ion, for example, the system water-hydrochloric acid-calcium perchlorate [6].

When, however, systems containing two salts with water as solvent are encountered, this emf method has only very limited application. In a few cases, suitable electrodes may be available; for example, the activity coefficient of zinc chloride in the presence of calcium chloride has been measured [7] using a cell with zinc amalgam and silver-silver chloride electrodes. Such well-behaved electrodes are not numerous and there are many interesting threecomponent systems where this emf method would be difficult, if not impossible, to apply. Thus it is difficult to see how suitable electrodes could be devised to study the very simple system watersodium chloride-potassium chloride and even more

\footnotetext{
${ }^{1}$ Figures in brackets indicate the literature references at the end of this paper.
}

difficult to suggest an emf method for the system water-sodium nitrate-potassium nitrate.

The isopiestic vapor pressure method has advantages in this respect and serves as a useful complement to the emf method. It has already been used to study the systems water-sodium chloride-potassium chloride [8] and water-potassium chloridepotassium bromide [9]. Results are now reported for the system water-sodium chloride-barium chloride; such results are of interest in themselves because they characterize a system of two simple inorganic solutes; in addition, this study reveals some difficulties which have to be overcome in extending the isopiestic method to solutions containing salts of different valence types.

\section{Methods}

\subsection{Definitions}

If an aqueous solution of sodium chloride is in isopiestic (vapor-phase) equilibrium with another aqueous solution containing both sodium chloride and barium chloride, then the two solutions have the same aqueous vapor pressure.

The following definitions are made.

$M_{B}$ is the molality (moles per kilogram of solvent) of a solution containing only sodium chloride (sometimes termed the "reference" solution).

$M_{c}$ is the molality of a solution containing barium chloride only, in isopiestic equilibrium with the sodium chloride solution of molality $M_{B}$.

$m_{B}, m_{c}$ are the molalities of sodium chloride and barium chloride, respectively, in a solution containing both these salts and in isopiestic equilibrium with the sodium chloride solution of molality $M_{B}$ and the barium chloride solution of molality $M_{c}$.

$R \equiv M_{B} /\left(m_{B}+1.5 m_{c}\right)$ is the isopiestic ratio.

$x \equiv 1.5 m_{c} /\left(m_{B}+1.5 m_{c}\right)$ is the "ionic fraction" of barium chloride in the mixture of sodium chloride and barium chloride. $m$ is any linear combination of the form

$$
m=k_{B} m_{B}+k_{c} m_{c}
$$


For this study it is convenient to put

$$
k_{B}=1, k_{c}=1.5, m=m_{B}+1.5 m_{c} .
$$

$\Gamma_{B}$ is the (molal) activity coefficient of sodium chloride in the reference solution at molality $M_{B}$ in equilibrium with the solution containing both sodium chloride and barium chloride.

$\Gamma_{c}$ is the activity coefficient of barium chloride at molality $M_{c}$ in equilibrium with the reference solution of sodium chloride at molality $M_{B}$.

$\gamma_{B}, \gamma_{c}$ are the activity coefficients of sodium chloride and barium chloride, respectively, in the mixed solution at molalities $m_{B}$ and $m_{c}$.

$\gamma_{B}^{\circ}$ is the activity coefficient of sodium chloride in a solution containing sodium chloride only at the same total ionic strength, $I=m_{B}+3 m_{c}$, as the mixed solution. $\gamma_{c}^{\circ}$ is the activity coefficient of barium chloride in a solution of barium chloride only at the same total ionic strength, $I=m_{B}+3 m_{c}$, as the mixed solution.

$\varphi$ is the osmotic coefficient of the reference solution, defined by the equation

$$
2 M_{B} \varphi=-55.51 \ln a_{w},
$$

$a_{w}$ being the water activity of this solution.

The distinction between $\Gamma_{B}$ and $\gamma_{B}^{\circ}$ and between $\Gamma_{c}$ and $\gamma_{c}^{\circ}$ is important. This somewhat complicated, but unavoidable, terminology is illustrated schematically in figure 1. The primary result of an isopiestic

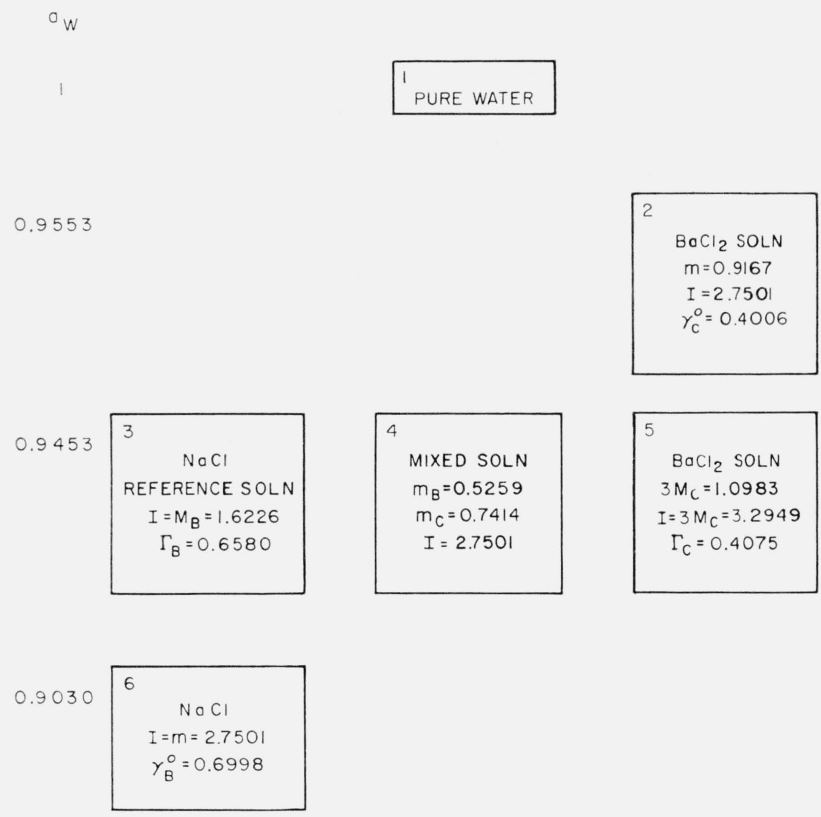

Figure 1.- Schematic representation of the system watersodium chloride-barium chloride.

The solutions represented by boxes 3,4 , and 5 are in isopiestic equilibrium, having the same water activity. The solutions reoresented by boxes 2,4 , and 6 are not in isopiestic equilibrium but have the same total ionic strength. $M_{B}$, $M_{C}, \gamma_{B}, \Gamma_{c}$ are known, as are $m_{B}$ and $m_{c} ; \gamma_{B}$ and $\gamma_{c}$, the activity coefficients of by box 4 are to be deduced. experiment is the molality $M_{B}$ of a sodium chloride solution (box 3 ) whose water activity $a_{w}$ and activity coefficient $\Gamma_{B}$ are known. This solution is in vaporphase equilibrium with a barium chloride solution (box 5) at molality $M_{c}$, the activity coefficient of barium chloride in this solution, $\Gamma_{c}$, also being known. These two solutions are also in vapor-phase equilibrium with a mixed solution of sodium chloride and barium chloride (box 4) of molalities $m_{B}$ and $m_{c}$, respectively. What are not known are the activity coefficients, $\gamma_{B}$ and $\gamma_{c}$, of sodium chloride and barium chloride, respectively, in this mixed solution, but it will be shown in the next section that the information in these three boxes is sufficient to calculate $\gamma_{B}$ and $\gamma_{c}$.

One may now enquire how $\gamma_{B}$ is related to $\gamma_{B}^{\circ}$; in particular, is what is sometimes called Harned's rule $[1,2]$ obeyed? An equation can be written:

$$
\log \gamma_{B}=\log \gamma_{B}^{\circ}-\alpha_{B} I_{c}-\beta_{B} I_{c}^{2}
$$

where $I_{c}=3 m_{c}$ and $\alpha_{B}, \beta_{B}$, are functions of the total ionic strength but not of the individual sodium chloride and barium chloride concentrations. Harned's Rule is equivalent to the statement that $\beta_{B}=0$. In order to investigate this, a knowledge of $\gamma_{B}^{\circ}$ is needed (box 6); that is to say we need to know the activity coefficient of a solution of sodium chloride only at the same total ionic strength as the mixed solution represented by box 4 in figure 1 . Similarly, for the other component:

$$
\log \gamma_{c}=\log \gamma_{c}^{\circ}-\alpha_{c} I_{B}-\beta_{c} I_{B}^{2}
$$

where $I_{B}=m_{B}$, and a knowledge of $\gamma_{c}^{\circ}($ box 2$)$ is needed to represent the variation of $\log \gamma_{c}$ with $I_{B}$.

\subsection{McKay-Perring Method}

McKay and Perring [10] have, using strict thermodynamic principles, derived an equation which can be put in the form:

$$
\ln \gamma_{B}=\ln \Gamma_{B}+\ln R+\int_{0}^{M_{B} \varphi} f\left(m, M_{B}, x\right) d\left(M_{B} \varphi\right)
$$

where

$$
f\left(m, M_{B}, x\right)=\frac{1}{m^{2}}\left(\frac{\partial m}{\partial \ln x}\right)_{a_{w}}+\frac{1}{m}-\frac{1}{M_{B}} .
$$

The isopiestic ratio, $R$, is the immediate result of the experiment; it is in the evaluation of the integral in eq (3) that difficulty is encountered. Let the reference solution be equilibrated, not with one mixed solution as illustrated in figure 1 , but with a series of mixed solutions of sodium chloride and barium chloride in different proportions, i.e., with different values of $x$, all the solutions having the same value of $a_{w}$. The quantity in the integral,

$$
\frac{1}{m^{2}}\left(\frac{\partial m}{\partial \ln x}\right)_{a_{w}}=-\frac{x}{M_{B}}\left(\frac{\partial R}{\partial x}\right)_{a_{w}}
$$


is determined by the tangent to the curve of $R$ versus $x$ for this particular water activity; this tangent will, in general, be a function of $x$ and the tangent is to be taken at that value of $x$ corresponding to which it is desired to evaluate the activity coefficients $\gamma_{B}$ and $\gamma_{c}$. The experiment must then be repeated to that $(\partial R / \partial x)$, at this particular value of $x$, can be determined as a function of $\left(M_{B} \varphi\right)$ and the integration over the interval 0 to $\left(M_{B} \varphi\right)$ performed. There is no theoretical way of predicting the variation of $(\partial R / \partial x)$ with $\left(M_{B} \varphi\right)$ but a considerable simplification ensues if, for the system in question, the isopiestic ratio can be expressed as

$$
R=a-b x-c x^{2}
$$

where $a, b$, and $c$ are functions of $M_{B}$ but not of $x$; for, in this case, the integral reduces to [8]

$$
x^{2} \int_{0}^{M_{B \varphi}}\left(c / M_{B}\right) d\left(M_{B} \varphi\right)
$$

and the integral vanishes if $c=0$,

$$
R=a-b x .
$$

In this case, eq (3) takes the very simple form

$$
\log \gamma_{B}=\log \Gamma_{B}+\log R
$$

and for the other component

where

$$
\log \gamma_{c}=\log \Gamma_{c}+\log R^{\prime}
$$

$$
R^{\prime}=\frac{1.5 M_{c}}{m_{B}+1.5 m_{c}} .
$$

\subsection{Experimental Procedure}

The apparatus was that used in an earlier investigation of the system water-sodium chloride-urea [11]. Eleven gold-plated silver dishes were used; three of them contained the reference sodium chloride solution and the remaining eight dishes were divided into four groups with four different mixtures each in duplicate (or three different mixtures and one pair containing a solution of barium chloride only). The sodium chloride was part of the sample used previously [11]; barium chloride was recrystallized twice from water. Analysis showed the presence of $25 \mathrm{ppm}$ of calcium and $350 \mathrm{ppm}$ of strontium. ${ }^{2}$ In each run, stock solutions of sodium chloride and barium chloride were prepared and mixed in different proportions. The stock solution of barium chloride was analyzed by the method of Newton and Tippetts [12].

The results of one experiment are quoted in detail to illustrate the agreement between the molalities of solutions in duplicate (or triplicate) dishes:

${ }^{2}$ Analyses by R. W. Burke of the Analysis and Purification Section.

\begin{tabular}{l|l|r|r|r|l}
\hline$m_{B}$ & 2.6189 & 1.7265 & 1.0075 & 0.2911 & 0 \\
& 2.6183 & 1.7261 & 1.0071 & .2911 & 0 \\
& 2.6182 & -1.2 & & & \\
$m_{c}$ & 0 & 0.5781 & 1.0450 & 1.5105 & 1.6998 \\
& 0 & .5780 & 1.0445 & 1.5107 & 1.6998 \\
\hline
\end{tabular}

\section{Results}

\begin{tabular}{|c|c|c|c|c|c|c|c|c|}
\hline Set & $M_{B}$ & $m_{B}$ & $m_{c}$ & $x$ & $R$ (obs.) & $R$ (calc.) & $\Delta_{1}$ & $\Delta_{2}$ \\
\hline 1 & .5063 & $\begin{array}{r}0.3591 \\
.2213 \\
.0794 \\
\\
.4033 \\
.2797 \\
.1453\end{array}$ & $\begin{array}{r}0.1045 \\
.2037 \\
.3060 \\
.3638 \\
.0736 \\
.1628 \\
.2597 \\
.3645\end{array}$ & $\begin{array}{r}0.3038 \\
.5800 \\
.8526 \\
1.0 \\
0.2149 \\
.4661 \\
.7283 \\
1.0\end{array}$ & $\begin{array}{r}0.9780 \\
.9576 \\
.9368 \\
.9244 \\
.9855 \\
.9666 \\
.9465 \\
.9248\end{array}$ & $\begin{array}{r}0.9787 \\
.9574 \\
.9364 \\
.9250 \\
.9855 \\
.9662 \\
.9460 \\
.9250\end{array}$ & $\begin{array}{r}+0.07 \\
-.02 \\
-.04 \\
+.06 \\
0 \\
-0.04 \\
-.05 \\
+.02\end{array}$ & $\begin{array}{c}+0.06 \\
+.04 \\
-.04 \\
0 \\
-.04 \\
+.01 \\
-.01 \\
-.04\end{array}$ \\
\hline 2 & .9728 & $\begin{array}{r}0.7382 \\
.4910 \\
.2610\end{array}$ & $\begin{array}{r}0.1644 \\
.3378 \\
.4999 \\
.6832\end{array}$ & $\begin{array}{l}0.2504 \\
.5079 \\
.7418 \\
1.0\end{array}$ & $\begin{array}{r}0.9878 \\
.9750 \\
.9623 \\
.9493\end{array}$ & $\begin{array}{r}0.9880 \\
.9747 \\
.9626 \\
.9493\end{array}$ & $\begin{array}{c}+0.02 \\
+.03 \\
+.03 \\
0\end{array}$ & $\begin{array}{c}0 \\
-0.02 \\
+.04 \\
-.03\end{array}$ \\
\hline 3 & 1. 2328 & $\begin{array}{r}0.9479 \\
.6389 \\
.3356\end{array}$ & $\begin{array}{r}0.1963 \\
.4092 \\
.6193 \\
.8507\end{array}$ & $\begin{array}{r}0.2370 \\
.4900 \\
.7346 \\
1.0\end{array}$ & $\begin{array}{r}0.9923 \\
.9841 \\
.9749 \\
.9661\end{array}$ & $\begin{array}{r}0.9924 \\
.9836 \\
.9752 \\
.9660\end{array}$ & $\begin{array}{r}+0.01 \\
-.05 \\
+.03 \\
-.01\end{array}$ & $\begin{array}{c}0 \\
-0.02 \\
+.05 \\
-.02\end{array}$ \\
\hline 4 & 1. 5683 & $\begin{array}{r}1.1653 \\
0.7442 \\
.2721\end{array}$ & $\begin{array}{r}0.2730 \\
.5586 \\
.8790 \\
1.0634\end{array}$ & $\begin{array}{r}0.2600 \\
.5297 \\
.8290 \\
1.0\end{array}$ & $\begin{array}{r}0.9959 \\
.9913 \\
.9860 \\
.9832\end{array}$ & $\begin{array}{r}0.9959 \\
.9913 \\
.9861 \\
.9832\end{array}$ & $\begin{aligned} & 0 \\
& 0 \\
&+ 0.01 \\
& 0\end{aligned}$ & $\begin{array}{l}-.01 \\
+.01 \\
+.01 \\
-.02\end{array}$ \\
\hline 5 & 1. 6226 & $\begin{array}{r}1.4141 \\
0.9784 \\
.5259\end{array}$ & $\begin{array}{r}0.1421 \\
.4356 \\
.7414 \\
1.0983\end{array}$ & $\begin{array}{r}0.1311 \\
.4005 \\
.6790 \\
1.0\end{array}$ & $\begin{array}{r}0.9972 \\
.9944 \\
.9906 \\
.9849\end{array}$ & $\begin{array}{r}0.9978 \\
.9939 \\
.9899 \\
.9853\end{array}$ & $\begin{array}{r}+0.06 \\
-.05 \\
-.07 \\
+.04\end{array}$ & $\begin{array}{r}+0.08 \\
-.04 \\
-.07 \\
+.03\end{array}$ \\
\hline 6 & 1.8749 & $\begin{array}{l}1.3930 \\
1.0035 \\
0.4308\end{array}$ & $\begin{array}{r}0.3219 \\
.5821 \\
.9647 \\
1.2498\end{array}$ & $\begin{array}{l}0.2574 \\
.4653 \\
.7706 \\
1.0\end{array}$ & $\begin{array}{r}0.9995 \\
.9990 \\
.9985 \\
1.0001\end{array}$ & $\begin{array}{r}0.9991 \\
.9992 \\
.9994 \\
.9995\end{array}$ & $\begin{array}{r}-0.04 \\
+.02 \\
+.09 \\
-.06\end{array}$ & $\begin{array}{r}-0.01 \\
+.02 \\
+.07 \\
-.06\end{array}$ \\
\hline 7 & 2. 0021 & $\begin{array}{r}1.2466 \\
0.7037 \\
.3073 \\
\\
1.5471 \\
1.0496 \\
0.5733\end{array}$ & $\begin{array}{r}0.4905 \\
.8501 \\
1.1124 \\
1.3149 \\
0.3006 \\
.6303 \\
.9452 \\
1.3245\end{array}$ & $\begin{array}{r}0.3712 \\
.6444 \\
.8445 \\
1.0 \\
0.2257 \\
.4739 \\
.7121 \\
1.0\end{array}$ & $\begin{array}{l}\text { 1. } 0025 \\
\text { 1. } 0042 \\
\text { 1. } 0058 \\
\text { 1. } 0076 \\
\text { 1. } 0021 \\
\text { 1. } 0035 \\
1.0055 \\
1.0077\end{array}$ & $\begin{array}{l}1.0027 \\
1.0048 \\
1.0063 \\
1.0074 \\
1.0017 \\
1.0035 \\
1.0053 \\
1.0074\end{array}$ & $\begin{array}{r}+0.02 \\
+.06 \\
+.05 \\
-.02 \\
-.04 \\
-0 \\
-0.02 \\
-.03\end{array}$ & $\begin{array}{r}+0.04 \\
+.06 \\
+.04 \\
-.04 \\
-.03 \\
+.01 \\
-.02 \\
-.05\end{array}$ \\
\hline 8 & 2. 1980 & $\begin{array}{r}1.8885 \\
1.3509 \\
0.7850 \\
.3184 \\
1.0177 \\
0.7602 \\
.4557 \\
.3008 \\
1.9192 \\
1.3574 \\
0.8234 \\
.3348\end{array}$ & $\begin{array}{r}0.1878 \\
.5407 \\
.9136 \\
1.2206 \\
0.7670 \\
.9375 \\
1.1377 \\
1.2396 \\
0.1845 \\
.5542 \\
.9060 \\
1.2274\end{array}$ & $\begin{array}{l}0.1298 \\
.3752 \\
.6358 \\
.8519 \\
.5306 \\
.6491 \\
.7892 \\
.8607 \\
.1260 \\
.3798 \\
.6227 \\
.8461\end{array}$ & $\begin{array}{l}1.0010 \\
1.0048 \\
1.0079 \\
1.0107 \\
1.0072 \\
1.0081 \\
1.0100 \\
1.0110 \\
1.0010 \\
1.0042 \\
1.0071 \\
1.0102\end{array}$ & $\begin{array}{l}1.0011 \\
1.0044 \\
1.0078 \\
1.0106 \\
1.0064 \\
1.0080 \\
1.0098 \\
1.0108 \\
1.0011 \\
1.0044 \\
1.0076 \\
1.0106\end{array}$ & $\begin{array}{l}+.01 \\
-.04 \\
-.01 \\
-.01 \\
-.08 \\
-.01 \\
-.02 \\
-.02 \\
+.01 \\
+.02 \\
+0.5 \\
+.04\end{array}$ & $\begin{array}{l}+.02 \\
-.10 \\
-.02 \\
+.06 \\
-.11 \\
-.02 \\
+.02 \\
+.05 \\
+.02 \\
-.02 \\
+.04 \\
+.10\end{array}$ \\
\hline 9 & 2.3615 & $\begin{array}{l}1.7350 \\
1.1799 \\
0.5922\end{array}$ & $\begin{array}{r}0.4108 \\
.7744 \\
1.1591 \\
1.5466\end{array}$ & $\begin{array}{l}0.2621 \\
.4961 \\
.7459 \\
1.0\end{array}$ & $\begin{array}{l}\text { 1. } 0044 \\
\text { 1. } 0085 \\
\text { 1. } 0131 \\
1.0179\end{array}$ & $\begin{array}{l}1.0043 \\
1.0086 \\
1.0131 \\
1.0178\end{array}$ & $\begin{array}{c}-0.01 \\
+.01 \\
0 \\
-0.01\end{array}$ & $\begin{array}{l}0 \\
0 \\
0 \\
0\end{array}$ \\
\hline 10 & 2. 6185 & $\begin{array}{l}\text { 1. } 7263 \\
\text { 1. } 0073 \\
0.2911\end{array}$ & $\begin{array}{l}\text { 0. } 5781 \\
1.0448 \\
1.5106 \\
1.6998\end{array}$ & $\begin{array}{l}0.5343 \\
.6087 \\
.8862 \\
1.0\end{array}$ & $\begin{array}{l}\text { 1. } 0097 \\
1.0171 \\
1.0241 \\
1.0270\end{array}$ & $\begin{array}{l}1.0098 \\
1.0170 \\
1.0241 \\
1.0271\end{array}$ & $\begin{array}{c}+0.01 \\
-.01 \\
0 \\
+0.01\end{array}$ & $\begin{array}{c}0 \\
0 \\
+0.01 \\
-.01\end{array}$ \\
\hline 11 & 2. 7147 & $\begin{array}{l}\text { 2. } 0250 \\
\text { 1. } 3969 \\
0.6356\end{array}$ & $\begin{array}{r}0.4451 \\
.8514 \\
1.3458 \\
1.7610\end{array}$ & $\begin{array}{r}\text { 0. } 2480 \\
.4776 \\
.7605 \\
1.0\end{array}$ & $\begin{array}{l}\text { 1. } 0082 \\
1.0152 \\
1.0228 \\
1.0277\end{array}$ & $\begin{array}{l}1.0087 \\
1.0147 \\
1.0221 \\
1.0283\end{array}$ & $\begin{array}{l}+.05 \\
-.05 \\
-.07 \\
+.06\end{array}$ & $\begin{array}{c}0 \\
-0.01 \\
-.03 \\
+.03\end{array}$ \\
\hline
\end{tabular}

The experimental results are given in table 1 . The second column gives the molality $\left(M_{B}\right)$ of the reference sodium chloride solution, the third and

TABLE 1. Isopiestic data for the system: water-sodium chloride $(B)$-barium chloride $(C)$ at $25{ }^{\circ} \mathrm{C}$

$\Delta_{1}=\{R($ calc by eq $(6))-R$ (obs) $] \times 10^{2}$.

$\Delta_{2}=[R($ calc by eq $(5))-R($ obs $)] \times 10^{2}$ 
fourth columns give values of the molalities of sodium chloride $\left(m_{B}\right)$ and barium chloride $\left(m_{c}\right)$ in the mixed solutions which were found to be in isopiestic equilibrium with the reference sodium chloride solution. The next column gives values of the "ionic fraction" $(x)$ and the sixth values of the isopiestic ratio. Figure 2 is a plot of isopiestic ratio versus ionic fraction for 3 of the 11 sets of results obtained; from this plot it would appear that $R$ is a linear function of $x$. The data in each set were therefore fitted to eq (7) and the $a$ and $b$ parameters evaluated by the method of least squares. These values of $a$ and $b$ are given in the second and third columns of table 2 and the values of $R$ calculated by eq (7) are given in the seventh column of table 1. The next column gives the difference (multiplied by 100 ) between the observed values of $R$ and those calculated by eq (7). The average of these differences for each set is given in the sixth column of table 2. It will be seen from figure 2 and from table 2 that the $a$ parameter does not have exactly the theoretical value of unity, suggesting that the plot of $R$ versus $x$ does have slight curvature, more pronounced at low values of $x$. The data were therefore fitted to eq (6) with $a=1$ and the $b$ and $c$ parameters, calculated by the method of least squares, are given in table 2 . Values of $R$ have been calculated with the aid of these parameters; they are not recorded in table 1 but the last column of this table gives the difference (multiplied by 100) between the observed values of $R$ and those calculated by eq (6); the average difference for each set is given in the last column of table 2 . It will be seen that any superiority eq (6) may have over eq (7) in representing the experimental data is within the experimental error; the simpler equation, (7), will therefore be used in subsequent calculations.

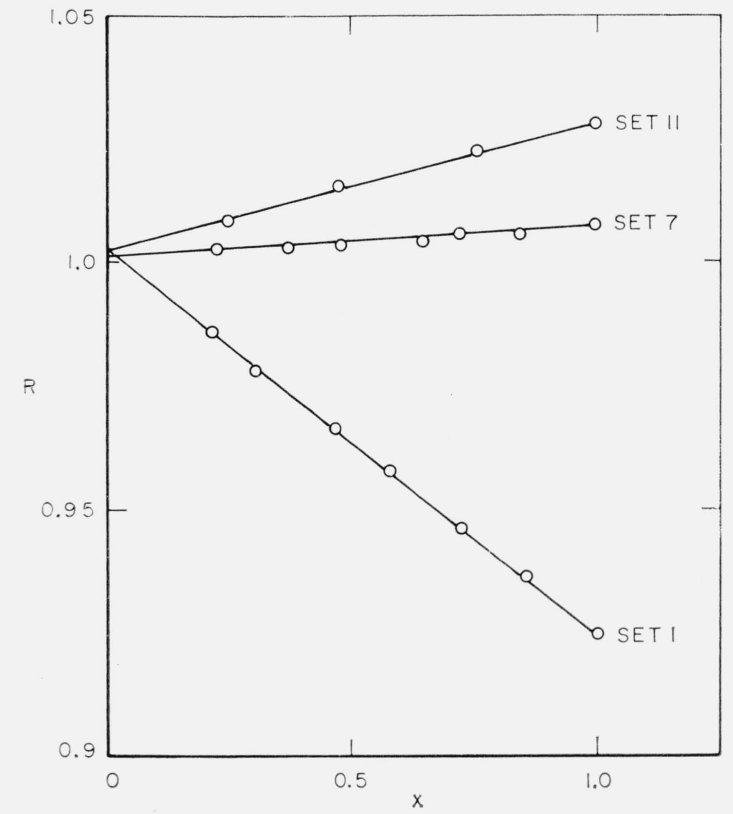

Figure 2. Isopiestic ratio $(R)$ versus ionic fraction $(x)$.
TABLE 2. Values of the parameters of eq (7) and eq (6)

\begin{tabular}{|c|c|c|c|c|c|c|}
\hline \multirow{2}{*}{ Set } & \multicolumn{2}{|c|}{$\mathrm{Eq}(7)$} & \multicolumn{2}{|c|}{$\operatorname{Eq}(6), a=1$} & \multirow{2}{*}{$\underset{\Delta_{1}}{\operatorname{Avg}}$} & \multirow{2}{*}{$\underset{\Delta_{2}}{A v g}$} \\
\hline & $a$ & $b$ & $b$ & $c$ & & \\
\hline 1 & 1.0021 & 0.0771 & 0.0681 & 0.0075 & 0.04 & 0.03 \\
\hline 2 & 1. 0009 & .0516 & .0481 & .0029 & .02 & .02 \\
\hline 3 & 1. 0006 & .0345 & 0318 & .0023 & .03 & .03 \\
\hline 4 & 1.0004 & .0172 & .0156 & 0014 & & .01 \\
\hline 5 & 0.9997 & .0144 & 0152 & -.0004 & 0.06 & .05 \\
\hline 6 & .9990 & .0006 & -.0030 & .0025 & .05 & .04 \\
\hline 7 & 1.0000 & -.0074 & -.0080 & .0008 & 03 & .04 \\
\hline 8 & 0.9994 & -.0132 & -.0085 & -.0056 & .04 & .05 \\
\hline 9 & .9995 & -.0183 & -.0164 & -.0015 & .01 & 0 \\
\hline 10 & 1.0012 & -.0259 & -.0300 & .0031 & .01 & 0.01 \\
\hline 11 & 1. 0023 & -.0260 & -.0349 & .0069 & .06 & .02 \\
\hline
\end{tabular}

\section{Calculation of Activity Coefficients}

This study of a mixture of a 1:1 electrolyte and a $2: 1$ electrolyte reveals a difficulty not encountered with mixtures of two electrolytes of the same valence type. This can be illustrated as follows. It was found [8] in a study of the system watersodium chloride-potassium chloride that the following solutions were in isopiestic equilibrium:

\begin{tabular}{r|l|l|l|l|l}
\hline \hline$m_{\mathrm{NaCl} 1}$ & 2.3248 & 1.8865 & 1.4490 & 1.0324 & 0.5630 \\
$m_{\mathrm{KC} 1}$ & 0 & 0.4894 & 0.9701 & 1.4238 & 1.9269 \\
$m_{\mathrm{NaC} 1}+m_{\mathrm{KC} 1}$ & 2.3248 & 2.3759 & 2.4191 & 2.4562 & 2.4899 \\
\hline
\end{tabular}

Thus only a small interpolation was needed to study the system at a total ionic strength of 2.4. It is very different with the system water-sodium chloridebarium chloride as the following example, taken from set 9 of table 1, shows:

\begin{tabular}{r|l|l|l|l|l}
\hline \hline$m_{B}$ & 2.3615 & 1.7350 & 1.1799 & 0.5922 & 0 \\
$m_{c}$ & 0 & 0.4108 & 0.7744 & 1.1591 & 1.5466 \\
$m_{B}+3 m_{c}$ & 2.3615 & 2.9574 & 3.5031 & 4.0695 & 4.6398 \\
$m_{B}+1.5 m_{c}$ & 2.3615 & 2.3512 & 2.3415 & 2.3309 & 2.3199 \\
\hline
\end{tabular}

Thus what remains approximately constant is not the total ionic strength $\left(m_{B}+3 \mathrm{~m}_{c}\right)$ but the "total ionic concentration" as measured by $\left(m_{B}+1.5 m_{c}\right)$; for, as the above table shows, the total ionic concentrations of the five isopiestic solutions are very nearly the same - the average value is 2.3410 and the largest deviation from this value is only 0.8 percent. We therefore considered whether Harned's Rule can hold in this form: the logarithm of the activity coefficient of sodium chloride is a linear function of the molality of barium chloride in mixtures of constant total ionic concentration, with a corresponding proposition for the activity coefficient of barium chloride. We therefore rewrite eq (1) and eq (2) as:

$$
\begin{aligned}
\log \gamma_{B} & =\log \gamma_{B}^{\circ}-\alpha_{B} m_{c} \\
\log \gamma_{c} & =\log \gamma_{c}^{\circ}-\alpha_{c} m_{B}
\end{aligned}
$$

$\gamma_{B}^{\circ}$ and $\gamma_{c}^{\circ}$ do not have the meanings assigned to 
them in section 2.1 ; in the present context, $\gamma_{B}^{\circ}$ is now the activity coefficient of sodium chloride in a solution of sodium chloride only at a molality equal to the ionic concentration $\left(m_{B}+1.5 m_{c}\right)$ of the mixed solution; $\gamma_{c}^{\circ}$ that of barium chloride in a solution of barium chloride only at a molality equal to $\left(m_{B}+1.5\right.$ $\left.m_{c}\right) / 1.5$. It would be possible to devise new symbols but there is no need to complicate the symbolism still further.

We now use eq (8) and eq (9) to calculate the activity coefficients of each salt in the mixture at constant total ionic concentration and then calculate $\alpha_{B}$ and $\alpha_{c}$ by eq (10) and eq (11). Values of $\gamma_{B}^{\circ}$ have already been tabulated [13]; data are available for barium chloride but the osmotic and activity coefficients have been computed again ${ }^{3}$ from earlier isopiestic data $(14,15]$ together with those results in table 1 which pertain to solutions of barium chloride without added sodium chloride. The data are given in table 3.

TABLE 3. Osmotic and activity coefficients of barium chloride in aqueous solution at $25^{\circ} \mathrm{C}$

\begin{tabular}{|c|c|c|c|c|c|}
\hline$m$ & $\varphi_{c}$ & $\gamma_{c}$ & $m$ & $\varphi_{c}$ & $\gamma_{c}$ \\
\hline 0.10 & 0.8422 & 0.5078 & 1.10 & 0.9520 & 0.4076 \\
\hline .20 & .8382 & .4523 & 1. 20 & .9681 & 4128 \\
\hline 30 & .8440 & .4265 & 1. 30 & .9845 & 4188 \\
\hline 40 & 8534 & 4121 & 1. 40 & 1. 0010 & 4255 \\
\hline .50 & 8648 & 4039 & 1. 50 & 1. 0175 & 4329 \\
\hline 60 & 8775 & 3996 & 1. 60 & 1. 0340 & 4408 \\
\hline .70 & .8912 & 3979 & 1. 70 & 1. 0503 & .4492 \\
\hline 80 & 9056 & 3982 & 1. 80 & 1. 0665 & 4580 \\
\hline .90 & 9206 & 4001 & 1.90 & 1. 0823 & 4672 \\
\hline 1. 00 & 9361 & 4033 & 2.00 & 1. 0978 & .4767 \\
\hline
\end{tabular}

Table 4 gives the values of $\alpha_{B}$ and $\alpha_{c}$. Substituted in eq (10) and eq (11), they will represent the data with a maximum difference of 0.0005 in $\log \gamma_{B}$ or $\log \gamma_{c}$, the average difference being much less than this. Within experimental error, therefore, it can be concluded that the proposed modification of Harned's Rule is valid for this system. Figure 3 is a plot of $\alpha_{B}$ and $\alpha_{c}$ versus $M_{B}$. This emphasizes the fact that $\alpha_{B}$ and $\alpha_{c}$ have large values in dilute solutions and small values in concentrated solutions. Thus there are large excess free energy terms in mixing dilute solutions of sodium chloride and barium chloride at constant total ionic concentration but these terms are much smaller (and of opposite sign) if the solutions are concentrated. This is a somewhat surprising result but confirms what has been observed before, particularly for the system water. hydrochloric acid-cesium chloride.

Table 4 also contains values of $\left(\alpha_{B}+1.5 \alpha_{c}\right)$ and these are plotted versus $M_{B}$ in figure $3 . \quad$ Now it has been shown [16, see also appendix] as a direct result of the cross-differentiation relation,

$$
2\left(\frac{\partial \log \gamma_{B}}{\partial m_{c}}\right)_{m_{B}}=3\left(\frac{\partial \log \gamma_{c}}{\partial m_{B}}\right)_{m_{c}}
$$

${ }^{3}$ We thank Dr. Wallace Davis, Jr., of the Oak Ridge National Laboratory for these computations. that the $\alpha_{B}$ and $\alpha_{c}$ parameters of eq (10) and eq (11) must obey the relation

$\left(\alpha_{B}+1.5 \alpha_{c}\right)=$ constant independent of $\left(m_{B}+1.5 m_{c}\right)$

provided that

(1) $\alpha_{B}$ and $\alpha_{c}$ are functions of $\left(m_{B}+1.5 \mathrm{~m}_{c}\right)$ only, and

(2) there are no terms in $m_{B}$ and $m_{c}$ other than $\alpha_{B} m_{c}$ and $\alpha_{c} m_{B}$, i.e., terms such as $\beta_{B} m_{c}^{2}$ are excluded. It will be seen that $\left(\alpha_{B}+1.5 \alpha_{c}\right)$ is very nearly constant, the largest deviation is at the lowest concentration where $\left(\alpha_{B}+1.5 \alpha_{c}\right)$ is the summation of two large terms of opposite sign. No significance is attached to the very slight variation of $\left(\alpha_{B}+1.5 \alpha_{c}\right)$ which is well within the experimental error and it is concluded that eq (10) and eq (11) suffice without the need for additional terms in higher powers of $m_{B}$ and $m_{c}$.

TABLE 4. Values of the parameters $\alpha_{B}$ and $\alpha_{\mathrm{c}}$ of eq (10) and $e q(11)$

\begin{tabular}{|c|c|c|c|}
\hline Set & $\alpha_{B}$ & $\alpha_{c}$ & $\left(\alpha_{B}+1.5 \alpha_{c}\right)$ \\
\hline $\begin{array}{r}1 \\
2 \\
3 \\
4 \\
5 \\
6 \\
7 \\
8 \\
9 \\
10 \\
11\end{array}$ & $\begin{array}{r}0.0859 \\
.0320 \\
.0175 \\
.0069 \\
.0060 \\
.0005 \\
-.0025 \\
-.0040 \\
-.0055 \\
-.0081 \\
-.0085\end{array}$ & $\begin{array}{r}-0.0602 \\
-.0225 \\
-.0128 \\
-.0047 \\
-.0046 \\
0 \\
.0019 \\
.0033 \\
.0044 \\
.0058 \\
.0055\end{array}$ & $\begin{array}{r}-0.0044 \\
-.0018 \\
-.0017 \\
-.0002 \\
-.0009 \\
.0005 \\
.0004 \\
.0010 \\
.0011 \\
.0006 \\
-.0002\end{array}$ \\
\hline
\end{tabular}

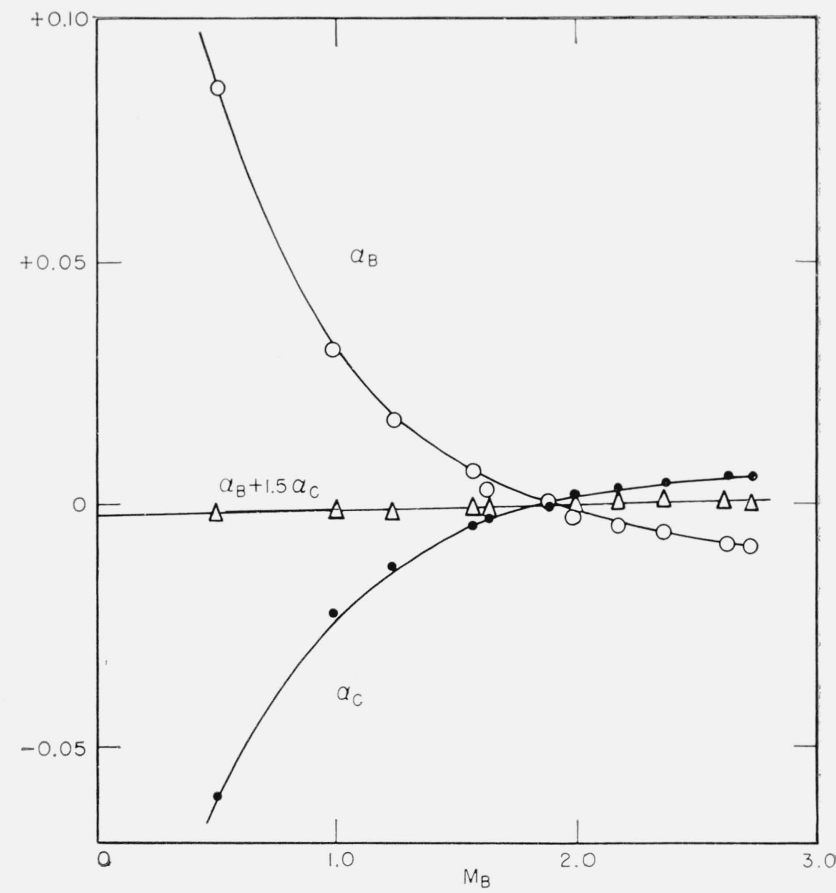

Figure 3. $\alpha_{B}, \alpha_{\mathrm{e}}$, and $\left(\alpha_{B}+1.5 \alpha_{c}\right)$ versus $M_{B}$ for systems at constant total ionic concentration. 


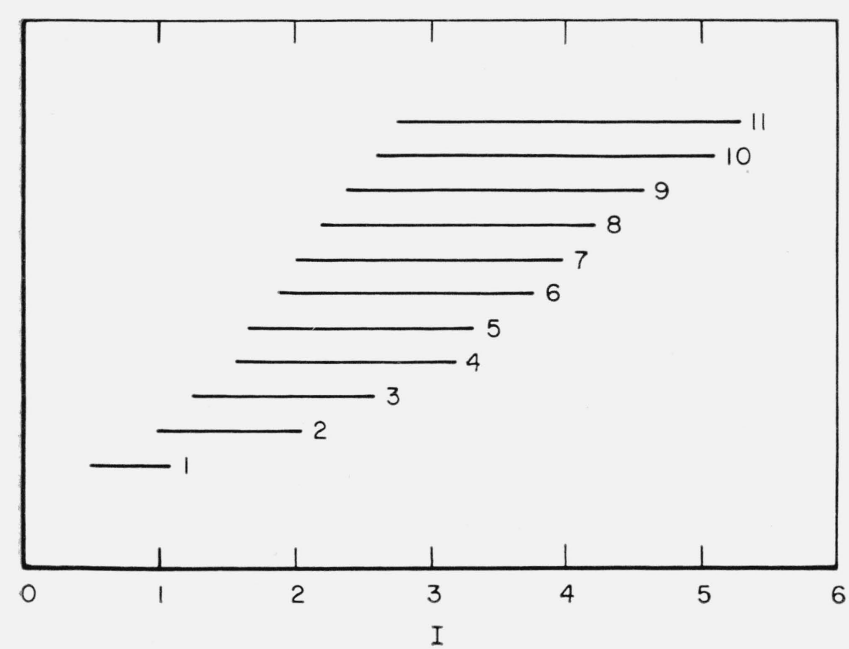

Figure 4. Span of total ionic strength in each set of measurements.

We could stop at this point because we have obtained a complete description of the system over the concentration range $0.5<\left(m_{B}+1.5 m_{c}\right)<2.7$; eq (10) and eq (11) with the $\alpha_{B}, \alpha_{c}$ parameters of table 4 give this information. It is of interest, however, to pursue the calculation and consider variations at constant total ionic strength instead of constant total ionic concentration. The difficulty of applying the isopiestic method to systems of different valence types at constant total ionic strength has already been referred to; figure 4 illustrates this in another way by showing the span of values of total ionic strength covered in each of the eleven sets of measurements. It shows that, whereas the set of five results quoted above for the system water-sodium chloride-potassium chloride contribute five points to the data at a total ionic strength, 2.4, any one set in the water-sodium chloride-barium chloride system contributes effectively only one point at any selected total ionic strength. Thus, results from different sets have to be harnessed in order to obtain data at different values of $x$ at any given total ionic strength. Moreover, the number of contributions depends on the total ionic strength. Thus, at $I=3$, sets 4 to 11 make eight contributions; at $I=5$, sets 10 and 11 only contribute.

We proceed as follows. At a total ionic strength of $I=3$, for example, we could have five solutions of the following compositions :

\begin{tabular}{c|c|c|c|c}
\hline \hline No. & $m_{B}=I_{B}$ & $I_{c}$ & $1.5 m_{c}$ & $m$ \\
& & & & \\
1 & 0.5 & 2.5 & 1.25 & 1.75 \\
2 & 1.0 & 2.0 & 1.00 & 2.00 \\
3 & 1.5 & 1.5 & 0.75 & 2.25 \\
4 & 2.0 & 1.0 & .50 & 2.50 \\
5 & 2.5 & 0.5 & .25 & 2.75 \\
\hline
\end{tabular}

A value of $\log \gamma_{B}$ for each solution can be calculated by eq (10) using the value of $\log \gamma_{B}^{\circ}$ appropriate to each value of $m$ and a value of $\alpha_{B}$ for each value of $m$ is read from a large scale plot of figure 3 . Then a new value of $\alpha_{B}$ is calculated by eq (1), ignoring the $\beta_{B} I_{c}^{2}$ terms. The calculation is as follows:

\begin{tabular}{c|r|r|r|r|r}
\hline \hline No. & $-\log \gamma_{B}^{\circ}$ & $\alpha_{B}$ eq (10) & $-\log \gamma_{B}$ & $\alpha_{B}$ eq (1) & $\begin{array}{c}-\log \gamma_{B} \\
\text { (calc. })\end{array}$ \\
\hline & & & & & \\
1 & 0.1799 & 0.0025 & 0.1820 & 0.0142 & 0.1818 \\
2 & .1755 & -.0018 & .1743 & .0139 & .1747 \\
3 & .1702 & -.0050 & .1677 & .0141 & .1677 \\
4 & .1627 & -.0070 & .1604 & .0139 & .1606 \\
5 & .1550 & -.0085 & .1531 & .0132 & .1536 \\
\hline
\end{tabular}

The best value of $\alpha_{B}$ for use in eq (1) is 0.0141 and, substituting for $-\log \gamma_{B}^{\circ}=0.1465$ at $I=3$, we get

$$
\log \gamma_{B}=-0.1465-0.0141 I_{c}
$$

Values of $-\log \gamma_{B}$ calculated by this equation are given in the last column of the above table; it is evident that, at least at $I=3$, eq (1) is adequate with only a term linear in $I_{c}$, so that $\beta_{B}=0$. Table 5

TABLE 5. Values of the parameters of eq (1) and eq (2)

\begin{tabular}{r|r|r|r}
\multicolumn{4}{c}{$\boldsymbol{\beta}_{B}=\boldsymbol{\beta}_{c}=0$} \\
\hline \hline$I$ & $\alpha_{B}$ & \multicolumn{1}{c}{$\boldsymbol{\alpha}_{c}$} & $\left(2 \alpha_{B}+\alpha_{c}\right)$ \\
\hline & & & \\
\hline 0.75 & 0.0082 & -0.0077 & 0.0087 \\
1.5 & .0095 & -.0085 & .0105 \\
2.0 & .0111 & -.0118 & .0104 \\
2.5 & .0127 & -.0155 & .0099 \\
3.0 & .0141 & -.0173 & .0109 \\
3.5 & .0159 & -.0191 & .0127 \\
4.0 & .0167 & -.0206 & .0128 \\
4.5 & .0178 & -.0218 & .0138 \\
5.0 & .0188 & -.0225 & .0151 \\
& & & \\
\hline
\end{tabular}

gives values of $\alpha_{B}$ and $\alpha_{c}$ calculated in this way at different values of the total ionic strength. The last column gives values of $\left(2 \alpha_{B}+\alpha_{C}\right)$, a quantity which is important because it can be shown [16, see also appendix] that in a system represented by eq (1) and eq (2) with $\beta_{B}=\beta_{c}=0$, the quantity $\left(2 \alpha_{B}+\alpha_{c}\right)$ should be independent of the total ionic strength. Not all the values of $\alpha_{B}$ and $\alpha_{c}$ in table 5 are of equal reliability, partly because at some values of $I$ we can use more sets of data from tables 1 and 4 than at others, and partly because there is some difficulty in interpolating in figure 5 at low values of $I$; the data at $I=2.5,3.0$, and 3.5 are probably more reliable, those at higher and lower total ionic strengths less so.

Figure 5 compares these values of $\alpha$ with those given by Harned and Gary $[1,4]$ for the system waterhydrochloric acid-barium chloride. We note that the $\alpha$ value for barium chloride is negative in both systems, so that the replacement of barium chloride by either hydrochloric acid or sodium chloride in a system at constant total ionic strength raises the activity coefficient of barium chloride; the effect is, however, much larger with hydrochloric acid. The $\alpha$ values for hydrochloric acid and sodium chloride are positive so that the activity coefficient of each is 


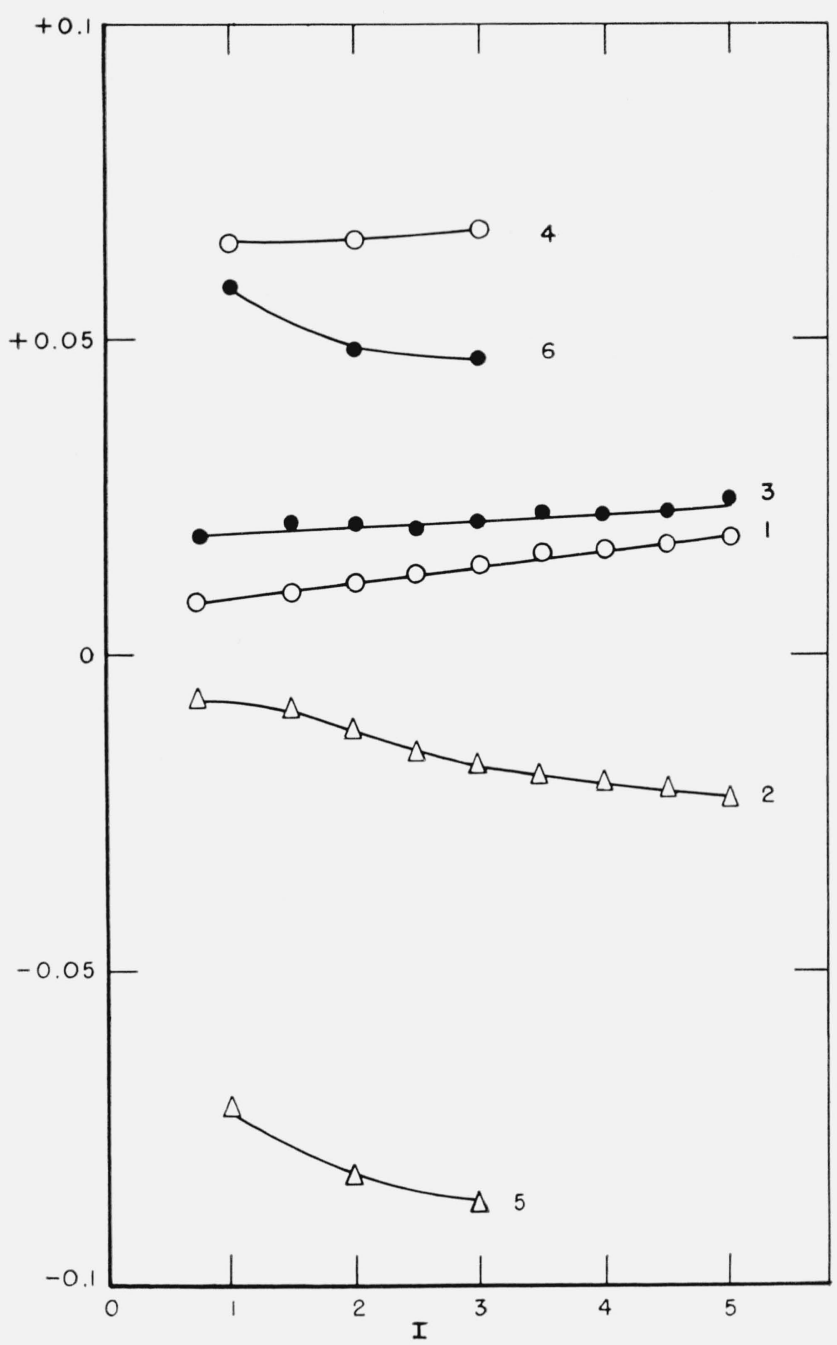

Figure 5. Parameters of eq (1) and eq (2).

System water; sodium chloride (B)-barium chloride (C) curve $1 \alpha_{B}$; curve $2 \alpha_{c}$; curve $3\left(2 \alpha_{B}+\alpha_{c}\right)$, the curve has been raised by 0.01 for the sake of clarity. System water-hydrochloric acid (B)-barium chloride (C) curve $4 \alpha_{B}$; curve $5 \alpha_{c}$; curve $6\left(2 \alpha_{B}+\alpha_{c}\right)$.

decreased by barium chloride; again the effect is very much larger with hydrochloric acid. For the sodium chloride-barium chloride system, $\left(2 \alpha_{B}+\alpha_{c}\right)$ increases slightly with increasing total ionic strength. The effect may be spurious, due to the difficulty we have in evaluating data in systems of constant total ionic strength. If the effect is real, it suggests that the $\beta$ terms in eq (1) and eq (2) are not both zero; $\beta_{B}=-0.0003, \beta_{c}=0$ would satisfy the condition demanded by the cross-differentiation relation, eq (12).

\section{Solubility Relations}

Let $m_{s}$ be the solubility of sodium chloride in water and $\gamma_{s}$ its activity coefficient at this molality.
Let $m_{B}$ be the solubility of sodium chloride in a solution of barium chloride of molality $m_{c}$ and $\gamma_{B}$ its activity coefficient. Then

or

$$
m_{s}^{2} \gamma_{s}^{2}=m_{B}\left(m_{B}+2 m_{c}\right) \gamma_{B}^{2}
$$

or

$$
m_{s} \gamma_{s}=m_{B}\left(1+2 m_{c} / m_{B}\right)^{1 / 2} \gamma_{B}
$$

$\log m_{B} / m_{s}=-\log \gamma_{B} / \gamma_{s}-1 / 2 \log \left(1+2 m_{c} / m_{B}\right)$.

Furthermore,

$$
\log \gamma_{B}=\log \gamma_{B}^{\circ}-3 \alpha_{B} m_{c}
$$

At $25{ }^{\circ} \mathrm{C}, m_{s}=6.147$ mole $\mathrm{kg}^{-1}$ [17] and $\log \gamma_{s}=$ 0.0020 . Moreover, in the vicinity of $m_{B}=6$ mole $\mathrm{kg}^{-1}, \log \gamma_{B}^{\circ}$ is almost linear in the total ionic strength,

$$
\log \gamma_{B}^{\circ}=\log \gamma_{s}+0.0545\left(m_{B}+3 m_{c}-m_{s}\right) .
$$

Hence

$\log m_{B} / m_{s}=-0.0545\left(m_{B}+3 m_{c}-m_{s}\right)+3 \alpha_{B} m_{c}$

$$
-1 / 2 \log \left(1+2 m_{c} / m_{B}\right) \text {. }
$$

Now $\alpha_{B}$ is a function of $\left(m_{B}+3 m_{c}\right)$ and $m_{B}$ is not known in advance. However, an inspection of table 5 and figure 5 shows that in concentrated solutions the value of $\alpha_{B}$ is not very sensitive to the value of the total ionic strength and for our present purpose we can put $\alpha_{B}=0.02$ as a good approximation. The solution of this equation predicts a solubility of 5.465 mole $\mathrm{kg}^{-1}$ in $0.5 \mathrm{~m}$ barium chloride solution and of 4.813 mole $\mathrm{kg}^{-1}$ in $1.0 \mathrm{~m}$ barium chloride solution (such solution would be supersaturated with respect to barium chloride).

The solubility of barium chloride in sodium chloride solutions is governed by somewhat different considerations because it is the dihydrate which is the stable solid phase. Representing the molality and activity coefficient of barium chloride in the saturated aqueous solution by $m_{s}$ and $\gamma_{s}$, respectively, we have:

$$
4 m_{s}^{3} \gamma_{s}^{3} a_{w(s)}^{2}=m_{c}\left(m_{B}+2 m_{c}\right)^{2} \gamma^{3} a_{v}^{2}
$$

where $a_{w}(s)$ is the water activity of the saturated aqueous solution and $a_{w}$ that of the saturated solution containing sodium chloride at molality $m_{B}$. Thus,

$\log m_{c} / m_{s}=-\log \gamma_{c} / \gamma_{s}-2 / 3 \log \left(1+m_{B} / 2 m_{c}\right)$

$$
-2 / 3 \log \left(a_{w} / a_{w(s)}\right) \text {. }
$$

At $25^{\circ} \mathrm{C}, m_{s}=1.790$ mole $\mathrm{kg}^{1-}[18], \log \gamma_{s}=-0.3400$. Again, $\log \gamma_{c}^{\circ}$ is almost linear in the total ionic strength:

$$
\log \gamma_{c}^{\circ}=\log \gamma_{s}+0.0283\left(m_{B}+3 m_{c}-3 m_{s}\right)
$$


so that

$\log m_{c} / m_{s}=-0.0283\left(m_{B}+3 m_{c}-3 m_{s}\right)+\alpha_{c} m_{B}$

$$
-\frac{2}{3} \log \left(1+m_{B} / 2 m_{c}\right)-\frac{2}{3} \log \left(a_{w} / a_{w(s)}\right) .
$$

From table 5 and figure 5 it would appear that $\alpha_{c} \approx-$ 0.023 for the saturated solution. In this way we have calculated solubilities of barium chloride of $1.401,1.045,0.736,0.484,0.299,0.175$, and 0.100 moles $/ \mathrm{kg}$ in solutions of $1.0,2.0,3.0,4.0,5.0,6.0$, and $7.0 \mathrm{~m}-\mathrm{NaCl}$, respectively. These data have been used to construct the two curves in figure 6 . There seem to be no direct measurements of mutual solubility at $25^{\circ}$ but Findlay and Cruickshank [19] determined the compositions of eight saturated solutions at $20^{\circ} \mathrm{C}$; these are represented by closed circles in figure 6 . Schreinemakers and de Baat [20] made eight measurements at $30^{\circ} \mathrm{C}$ and these are represented by open circles in figure 6 . In spite of the approximations we made in calculating solubilities from isopiestic data, the agreement is good and confirms the validity of the data given in table 5 to describe the activity coefficients of the salts in the system water-sodium chloride-barium chloride.

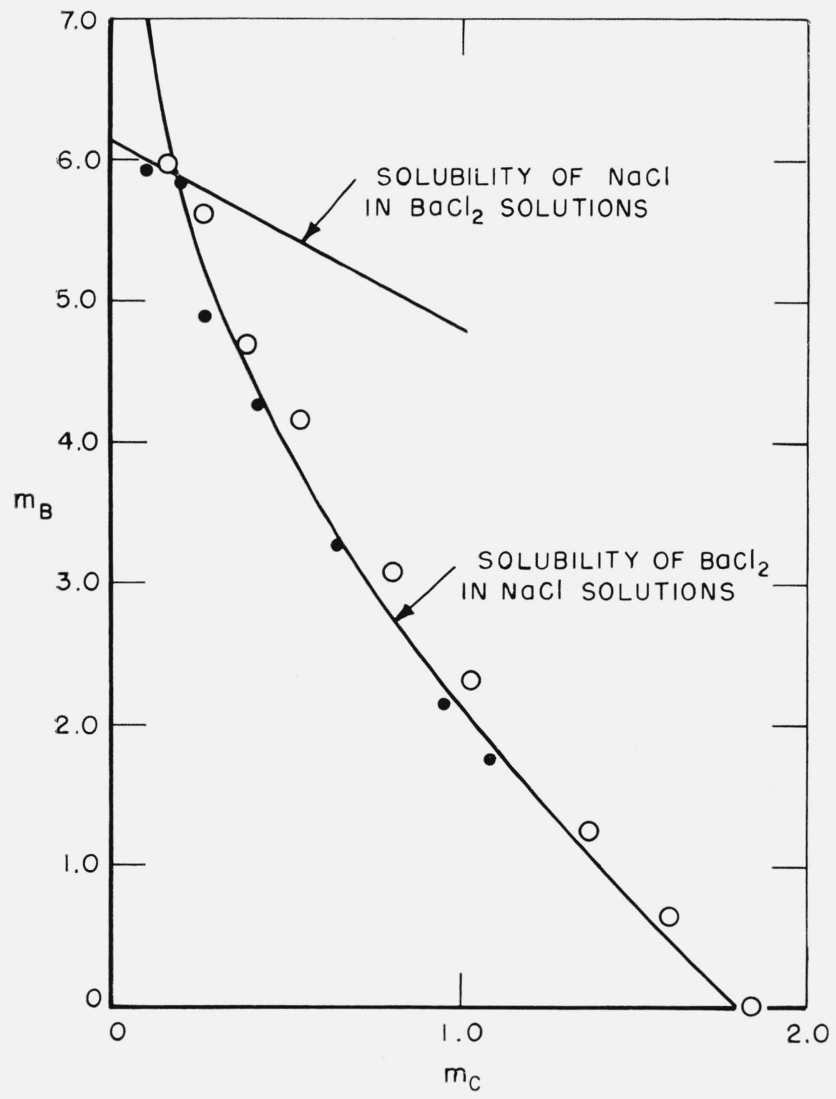

FIgURE 6. Solubility relations in the system water-sodium chloride-barium chloride.

- Solubility curves calculated from isopiestic data at $25^{\circ} \mathrm{C}$ , Direct measurements at $20^{\circ} \mathrm{C}$. , Direct measurements at $30^{\circ} \mathrm{C}$.

\section{Appendix}

Although the derivation of eq (13) has already been described [1, 16], it is worthwhile considering the more general case of a mixture of electrolytes of different valence types for which the $\beta$ terms of eq (1) and eq (2) are not zero. Consider a solution of electrolyte $B$ (giving $\nu_{B}$ ions) at molality $m_{B}$, and electrolyte $C$ (giving $\nu_{c}$ ions) at molality $m_{c}$. Let $\zeta$ be a linear combination of the form

$$
\zeta=k_{B} m_{B}+k_{c} m_{c}
$$

and let the activity coefficients at constant $\zeta$ be given by

$$
\begin{gathered}
\log \gamma_{B}=\log \gamma_{B}^{\circ}-A_{B} m_{c}-B_{B} m_{c}^{2} \\
\log \gamma_{c}=\log \gamma_{c}^{\circ}-A_{c} m_{B}-B_{c} m_{B}^{2}
\end{gathered}
$$

where $A_{B}$ and $A_{c}$ are functions of $\zeta$ but not of $m_{B}$ and $m_{c}$ individually and $B_{B}, B_{c}$ are parameters independent of $\zeta, m_{B}$, and $m_{c}$. The cross-differentiation relation:

$$
\nu_{B}\left(\frac{\partial \log \gamma_{B}}{\partial m_{c}}\right)_{m_{B}}=\nu_{c}\left(\frac{\partial \log \gamma_{c}}{\partial m_{B}}\right)_{m_{c}}
$$

can be written:

$$
\nu_{B} k_{c}\left(\frac{\partial \log \gamma_{B}}{\partial \zeta}\right)_{m_{B}}=\nu_{c} k_{B}\left(\frac{\partial \log \gamma_{c}}{\partial \zeta}\right)_{m_{c}}
$$

Moreover,

$\log \gamma_{B}=\log \gamma_{B}^{\circ}-A_{B}\left(\zeta-k_{B} m_{B}\right) / k_{c}-B_{B}\left(\zeta-k_{B} m_{B}\right)^{2} / k_{c}^{2}$

and

$$
\log \gamma_{c}=\log \gamma_{c}^{\circ}-A_{c}\left(\zeta-k_{c} m_{c}\right) / k_{B}-B_{c}\left(\zeta-k_{c} m_{c}\right)^{2} / k_{B}^{2}
$$

As $\log \gamma_{B}^{\circ}, \log \gamma_{c}^{\circ}, A_{B}$, and $A_{c}$ are functions of $\zeta$ only,

$$
\begin{gathered}
\nu_{B} k_{c}\left\{\frac{d \log \gamma_{B}^{\circ}}{d \zeta}-\frac{A_{B}}{k_{c}}-\frac{\zeta-k_{B} m_{B}}{k_{c}} \frac{d A_{B}}{d \zeta}-2 B_{B} \frac{\zeta-k_{B} m_{B}}{k_{c}^{2}}\right\} \\
=\nu_{c} k_{B}\left\{\frac{d \log \gamma_{c}^{\circ}}{d \zeta}-\frac{A_{c}}{k_{B}}-m_{B} \frac{d A_{c}}{d \zeta}-2 B_{c} m_{B} / k_{B}\right\} .
\end{gathered}
$$

If this equality is to hold for all values of $\zeta$ and $m_{B}$, the coefficient of $m_{B}$ must vanish, and

$$
\begin{aligned}
\nu_{B} k_{B} k_{c} \frac{d A_{B}}{d \zeta}+\nu_{c} k_{B} k_{c} \frac{d A_{c}}{d \zeta} \\
=-2\left(v_{B} k_{B} B_{B}+v_{c} k_{c} B_{c}\right)
\end{aligned}
$$$$
\text { or }
$$

$$
\begin{gathered}
k_{B} k_{c}\left(v_{B} A_{B}+v_{c} A_{c}\right)=\text { constant } \\
-2\left(v_{B} k_{B} B_{B}+v_{c} k_{c} B_{c}\right) \zeta
\end{gathered}
$$

If $v_{B}=2, v_{c}=3, k_{B}=1, k_{c}=3$, as with sodium chloride and barium chloride at constant total ionic strength, 
$\left(6 A_{B}+9 A_{c}\right)=\mathrm{constant}-2\left(2 B_{B}+9 B_{c}\right) I$ or, putting $A_{B}=3 \alpha_{B}, A_{c}=\alpha_{c}, B_{B}=9 \beta_{B}, B_{c}=\beta_{c}$,

$\log \gamma_{B}=\log \gamma_{B}^{\circ}-\alpha_{B} I_{c}-\beta_{B} I_{c}^{2}$

$\log \gamma_{c}=\log \gamma_{c}^{\circ}-\alpha_{c} I_{B}-\beta_{c} I_{B}^{2}$

which are eq (1) and eq (2), then

$$
\left(2 \alpha_{B}+\alpha_{c}\right)=\text { constant }-2\left(2 \beta_{B}+\beta_{c}\right) I .
$$

If $v_{B}=2, v_{c}=3, k_{B}=1, \quad k_{c}=1.5$, as with sodium chloride and barium chloride at constant total ionic concentration,

$1.5\left(2 A_{B}+3 A_{c}\right)=$ constant $-2\left(2 B_{B}+4.5 B_{c}\right) \quad\left(m_{B}+\right.$ $\left.1.5 m_{c}\right)$

or, putting $A_{B}=\alpha_{B}, A_{c}=\alpha_{c}, \beta_{B}=\beta_{c}=0$,

$$
\log \gamma_{B}=\log \gamma_{B}^{\circ}-\alpha_{B} m_{c},
$$$$
\log \gamma_{c}=\log \gamma_{c}^{\circ}-\alpha_{c} m_{B}
$$

which are eq (10) and eq (11), then

$$
\left(\alpha_{B}+1.5 \alpha_{c}\right)=\text { constant }
$$

Alternatively, we could put $A_{B}=1.5 \alpha_{B}$, $A_{c}=\alpha_{c}, B_{B}=1.5^{2} \beta_{B}, B_{c}=\beta_{c}$, so that:

$\log \gamma_{B}=\log \gamma_{B}^{\circ}-\alpha_{B}\left(1.5 m_{c}\right)-\beta_{B}\left(1.5 m_{c}\right)^{2}$,

$\log \gamma_{c}=\log \gamma_{c}^{\circ}-\alpha_{c} m_{B}-\beta_{c} m_{B}^{2}$

and obtain the more compact equation:

$\left(\alpha_{B}+\alpha_{c}\right)=$ constant $-2\left(\beta_{B}+\beta_{c}\right)\left(m_{B}+1.5 m_{c}\right)$.

\section{References}

[1] H. S. Harned and B. B. Owen, The physical chemistry of electrolytic solutions, ch. 14, 3d. Ed., (Reinhold Publ. Corp., New York, N.Y., 1958).

[2] R. A. Robinson and R. H. Stokes, Electrolvte solutions, ch. 15, 2d. Ed., (Butterworths Scientific Publications, London, 1959).

[3] H. S. Harned and T. R. Paxton, J. Phys. Chem. 57, 531 (1961).

[4] H. S. Harned and R. Gary, J. Am. Chem. Soc. 76,5924 (1954).

[5] W. J. Argersinger, L. Leifer, and A. W. Davidson, J. Phys. Chem. 5\%, 531 (1961).

[6] J. M. Stokes and R. H. Stokes, J. Phys. Chem 67, 2442 (1963)

[7] R. A. Robinson and R. O. Farrelly, J. Phys. Chem. 51, $704(1947)$.

[8] R. A. Robinson, J. Phys. Chem. 65, 662 (1961).

[9] W. H. McCoy and W. E. Wallace, J. Am. Chem. Soc. 78, 1830 (1956).

[10] H. A. C. MeKay and J. K. Perring, Trans. Faraday Soc. 49, 163 (1953).

[11] V. E. Bower and R. A. Robinson, J. Phys. Chem. 6y, 1524 (1963).

[12] E. A. Tippetts and R. F. Newton, J. Am. Chem. Soc. 56, 1675 (1934).

[13] Reference 2, p. 476.

[14] R. A. Robinson, Trans. Faraday Soc. 36, 735 (1940).

[15] R. A. Robinson, Proc. Roy. Soc. New Zealand 75, 203 (1955).

[16] E. Glueckauf, H. A. C. McKay, and A. R. Mathieson, J. Chem. Soc. (London) S299 (1949).

[17] F. J. Kelly, R. A. Robinson, and R. H. Stokes, J. Phys. Chem. 65, 1958 (1961).

[18] H. W. Foote and F. C. Hickey, J. Am. Chem. Soc. 59, 648 (1937)

[19] A. Findlay and J. Cruickshank, J. Chem. Soc. (London) 129, 316 (1926).

[20] F. A. H. Schreinemakers and W. C. de Baat, Z. Phys, Chem. 65, 586 (1908-1909).

(Paper 69A1-323) 